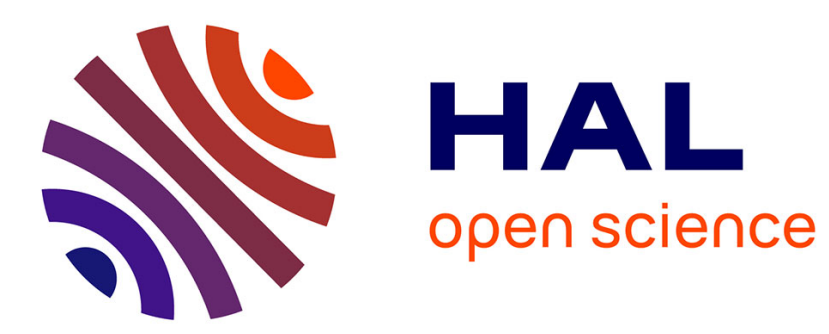

\title{
Traducteurs ultrasonores large-bande à profil vibratoire non uniforme. Théorie et expériences
}

\author{
A. Lhémery, D. de Vadder, S. Dimitriu
}

\section{To cite this version:}

A. Lhémery, D. de Vadder, S. Dimitriu. Traducteurs ultrasonores large-bande à profil vibratoire non uniforme. Théorie et expériences. Journal de Physique IV Proceedings, 1994, 04 (C5), pp.C5-1193C5-1196. 10.1051/jp4:19945263 . jpa-00252953

\section{HAL Id: jpa-00252953 https://hal.science/jpa-00252953}

Submitted on 1 Jan 1994

HAL is a multi-disciplinary open access archive for the deposit and dissemination of scientific research documents, whether they are published or not. The documents may come from teaching and research institutions in France or abroad, or from public or private research centers.
L'archive ouverte pluridisciplinaire HAL, est destinée au dépôt et à la diffusion de documents scientifiques de niveau recherche, publiés ou non, émanant des établissements d'enseignement et de recherche français ou étrangers, des laboratoires publics ou privés. 


\title{
Traducteurs ultrasonores large-bande à profil vibratoire non uniforme. Théorie et expériences
}

\author{
A. LHÉMERY, D. DE VADDER et S. DIMITRIU
}

Ecole Centrale Paris, Laboratoire de Mécanique, URA 850 du CNRS, Equipe Ultrasons, Grande Voie des Vignes, 92295 Châtenay-Malabry cedex, France

\begin{abstract}
This paper follows a strictly theoretical one presented in the previous CFA [1]. We had shown the advantage of using nonuniformly excited transducers in wideband pulse-echo method for nondestructive tetsing. We can now produce the source (and sensitivity) profile we want by a masking method not detailed herein. We limit the study to planar disc transducers. The transient field radiated by a planar transducer vibrating with a conventional uniform profile is often described as a superposition of a plane wave and edge (diffracted) waves. We consider three different profiles: uniform profile, profile favoring the plane wave component and another favoring the edge wave. We first compare theoretical with measured echo-responses from simple-shaped targets proving the validity of the masking technique. Then, practical transducers are studied in terms of their axial or radial resolution and sensitivity.
\end{abstract}

\section{INTRODUCTION.}

La structure multiple des échos ultrasonores revenant de cibles (même ponctuelles [2]) dans le champ proche d'un traducteur large bande peut conduire à des ambiguïtés d'interprétation. De plus, la forme des échos est fortement dépendante de la distance tant que la cible n'est pas dans le champ lointain. Nous avions montré théoriquement [1] l'influence du profil de source (et de sensibilité) du traducteur sur cette structure en illustrant nos propos par quelques exemples de simulation. Nous savons désormais fabriquer par une méthode de masquage des traducteurs à profil non-uniforme (la méthode, suggérée dans [3] pour les traducteurs bande étroite, n'est pas détaillée ici). Cet article montre dans un premier temps par comparaison de résultat de simulation et d'expériences que les profils produits sont conformes à ceux souhaités. Dans un second temps, les traducteurs fabriqués sont étudiés en terme de résolution axiale ou radiale. Enfin, l'aspect quantitatif (la sẹnsibilité réelle des traducteurs considérés) est abordé.

\section{COMPARAISON THÉORIE-EXPÉRIENCE.}

Un traducteur émettant dans un milieu fluide est modélisé comme une source de vitesse particulaire. Si les modes radiaux de vibrations sont négligeables, les dépendances spatiales et temporelles sont séparables dans le terme de source $v_{n}\left(\mathbf{r}_{R}, t\right)$, et $v_{n}\left(\mathbf{r}_{R}, t\right)=\Gamma\left(\mathbf{r}_{R}\right) v(t)$, où $\mathbf{r}_{R}$ est un point courant du traducteur. Les profils de source et de sensibilité (à la pression) $\Gamma\left(\mathbf{r}_{R}\right)$ sont identiques. A partir d'un traducteur uniforme circulaire standard vibrant en piston uniforme [profil $\Gamma_{1}\left(r_{R}\right)=1$ pour $r_{R} \leq a$ ], nous avons fabriqué deux traducteurs non-uniformes dont les profils $\Gamma_{2}\left(r_{R}\right)$ et $\Gamma_{3}\left(r_{R}\right)$ sont donnés par,

$$
\begin{aligned}
& \begin{cases}\Gamma_{2}\left(r_{R}\right)=1, & \text { pour } r_{R}<a_{0} \\
\Gamma_{2}\left(r_{R}\right)=\left\{\cos \left[\pi\left(r_{R}-a_{0}\right) /\left(a-a_{0}\right)\right]+1\right\} / 2, & \text { pour } a_{0} \leq r_{R} \leq a,\end{cases} \\
& \begin{cases}\Gamma_{3}\left(r_{R}\right)=0, & \text { pour } r_{R}<a_{0} \\
\Gamma_{3}\left(r_{R}\right)=\left\{1-\cos \left[\pi\left(r_{R}-a_{0}\right) /\left(a-a_{0}\right)\right]\right\} / 2, & \text { pour } a_{0} \leq r_{R} \leq a,\end{cases}
\end{aligned}
$$

avec $a=17 \mathrm{~mm}$ et $a_{0}=8 \mathrm{~mm} . \Gamma_{2}\left(r_{R}\right)$ et $\Gamma_{3}\left(r_{R}\right)$ sont complémentaires. Le champ rayonné par le profil uniforme est classiquement décrit [2] comme superposition d'une onde plane et d'ondes (diffractées) de bord. $\Gamma_{2}\left(r_{R}\right)$ favorise la composante plane du champ transitoire, $\Gamma_{3}\left(r_{R}\right)$ favorise l'onde de bord. Nous pouvons vérifier que le profil des traducteurs non-uniformes fabriqués correspond bien au profil théo- 
rique voulu donné par les Eqs. (1). Nous disposons pour cela d'un modèle [4] qui a été validé expérimentalement [5]. La Fig. 1 montre un exemple de comparaison de résultats théoriques et expérimentaux pour deux cibles disques. On constate un bon accord entre les mesures et les prédictions. L'accord est qualitatif et quantitatif, les échos prédits et mesurés étant représentés aux mêmes amplitudes relatives. On en conclut que les traducteurs se comportent essentiellement comme souhaités. On peut alors s'intéresser à la mise en évidence expérimentale de leur caractéristiques les plus intéressantes.
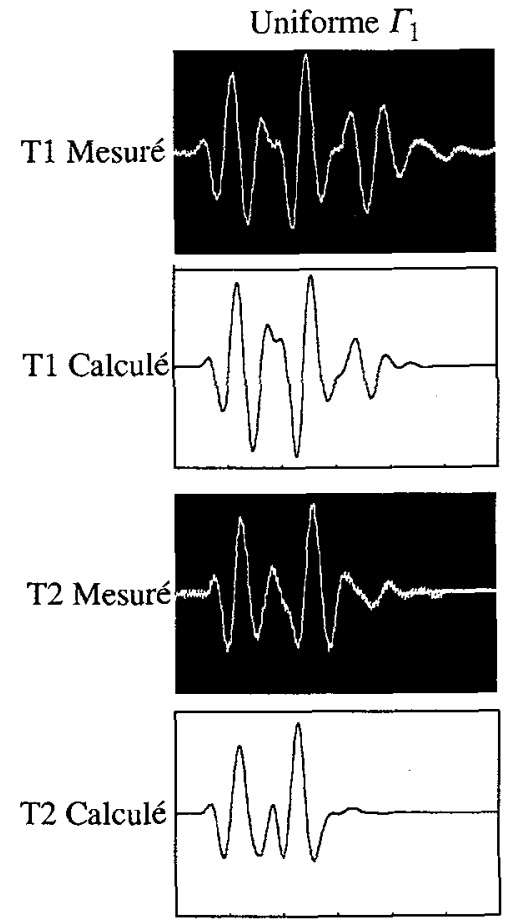

à onde plane $\Gamma_{2}$
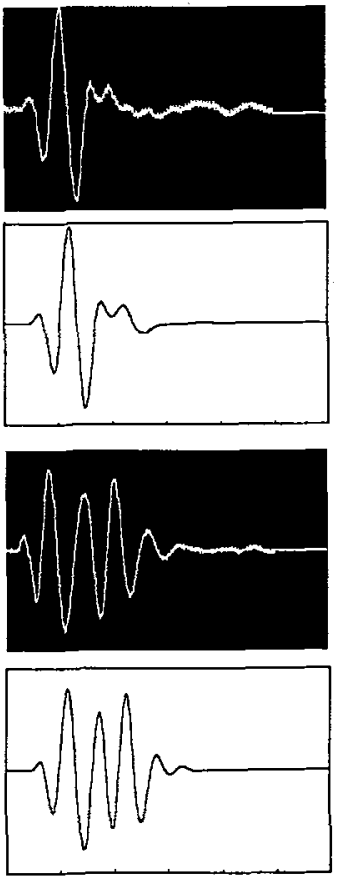

à onde de bord $\Gamma_{3}$
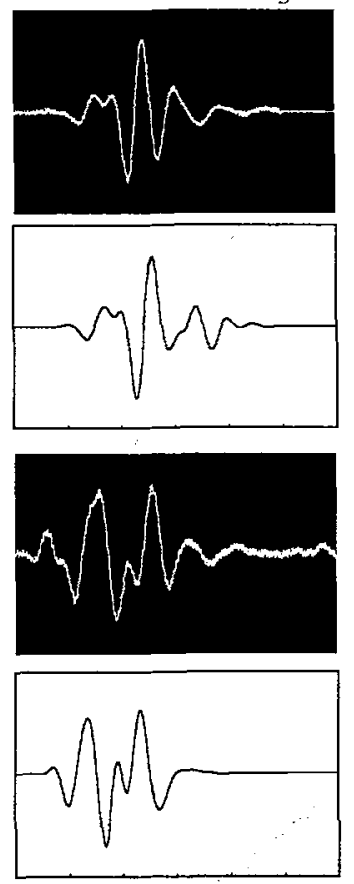

Fig. 1 - Comparaison expériences / simulations : T1, disque de 4-mm- $\varnothing$ à une distance de $35 \mathrm{~mm}$ du traducteur. T2, disque de $10-\mathrm{mm}-\emptyset$ à une distance de $120 \mathrm{~mm}$, l'un et l'autre inclinés de $10^{\circ}$ par rapport au traducteur (1 $\mu$ s / div.)

\section{RESOLUTION DES TRADUCTEURS NON-UNIFORMES.}

Les cibles utilisées pour les mesures de résolution sont constituées de deux réflecteurs disques (extrémité de cylindres) positionnés à des distances axiales et radiales différentes (par rapport au traducteur). La Fig. 2 donne leur forme typique.

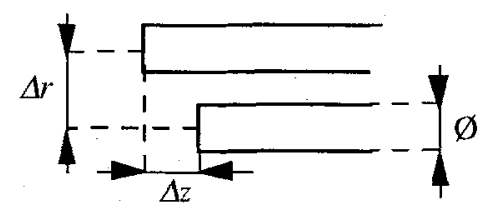

Fig. 2 - Type de cible utilisée pour la mesure de résolution des traducteurs. Trois paramètres: $\Delta r, \Delta z$ et $\varnothing$.

\subsection{Résolution axiale du traducteur à onde plane}

Du fait de la grande variation avec la distance de la forme des échos revenant d'une cible, la résolution axiale d'un traducteur plan uniforme ne devient correcte que dans le champ lointain. Le traducteur de profil $\Gamma_{2}$ favorisant la composante plane peut être considéré comme non-diffractant. La structure multiple des échos, caractéristique des effets de diffraction par le traducteur, se trouve simplifiée pour une cible dans le champ proche. La cible utilisée a pour paramètres; $\Delta r=1.5 \mathrm{~mm}, \Delta z=2 \mathrm{~mm}, \varnothing=1 \mathrm{~mm}$. La Fig. 3 montre les échos mesurés revenant de cette cible à différentes distances des traducteurs de profil $\Gamma_{1}$ et $\Gamma_{2}$. Avec le profil $\Gamma_{2}$ les deux réflecteurs sont résolus quelle que soit la distance. Avec le traducteur uniforme, ce n'est qu'une fois atteint le champ lointain que l'interprétation correcte des échos est possible. Dans le champ proche, les échos multiples revenant de chacun des deux réflecteurs interfèrent et rendent 
impossible l'interprétation. Il est à noter que tous les résultats sont représentés à la même échelle. Nous reviendrons sur l'aspect quantitatif dans le paragraphe 4.

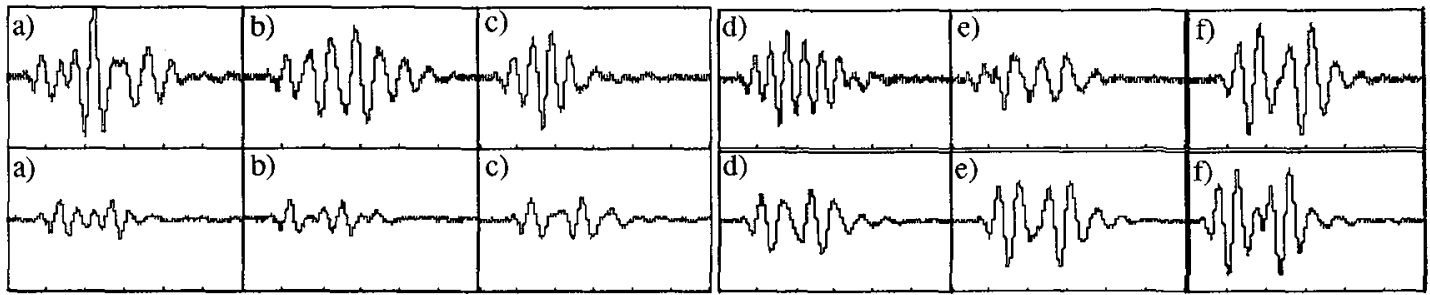

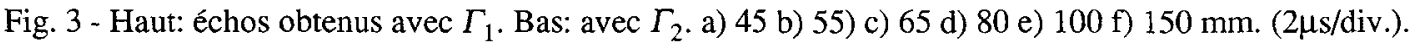

\subsection{Résolution radiale du traducteur à onde plane}

La résolution radiale d'un traducteur uniforme est mal définie en champ proche car rapidement variable d'un point à un autre. En supprimant l'effet de diffraction dans le champ proche, le profil $\Gamma_{2}$ rend la résolution constante mais celle-ci reste médiocre. L'étude précise de cette résolution n'est pas présentée ici.

\subsection{Résolution radiale du traducteur à onde de bord}

Les traducteurs annulaires sont connus pour leur très bonne résolution radiale [6]. Le traducteur de profil $\Gamma_{3}$ rayonne essentiellement comme un traducteur annulaire avec cependant une caractéristique différente: si la rupture entre la partie vibrante et non vibrante de la surface en $r_{R}=a$ est brusque dans $\Gamma_{3}$ comme pour un traducteur annulaire, le passage de la partie vibrante à la partie non-vibrante au centre est adoucie du fait de la variation en cosinus[Eq.(1b)], au lieu d'une rupture brusque pour le traducteur annulaire. Dans une exploration systématique du champ produit par ce traducteur, que le manque de place nous empêche de présenter ici, nous avons pu montrer que cette caractéristique spécifique au profil considéré atténue l'amplitude des lobes secondaires existant dans le cas d'un traducteur annulaire classique. Mais la caractéristique de bonne résolution radiale est maintenue. Il est dès lors plus approprié de comparer le traducteur de profil $\Gamma_{3}$ à un traducteur focalisé de même diamètre. Nous avons utilisé à nouveau une cible formée de deux disques avec les paramètres suivants: $\Delta r=3 \mathrm{~mm}, \Delta z=1.2 \mathrm{~mm}, \emptyset=1.2 \mathrm{~mm}$. Pour explorer la résolution radiale, nous avons relevé l'amplitude crête à crête des échos revenant de la cible comme une fonction du déplacement radial à une distance axiale $z$ donnée du traducteur, et répété la mesure à différentes distances $z$. La Fig. 4a) montre un relevé radial typique. Deux pics sont discernables. Ils correspondent au passage sur l'axe acoustique de chacun des deux réflecteurs. On mesure le creux de hauteur $h$ (en $\mathrm{dB}$ ) entre les deux pics, relativement au pic de plus petite amplitude. La Fig. 4 b) montre comment $h$ varie avec la distance $z$, $h$ peut atteindre $14 \mathrm{~dB}$ en champ proche. Un traducteur de diamètre $D$ est considéré comme "bien focalisant" si sa distance focale $F$ est plus petite que 0.6 fois la distance limite de champ lointain du traducteur plan de même diamètre, i.e., $F<0.6 D^{2} / 4 \lambda_{c}\left(\lambda_{c}=c / v_{c}, v_{c}=0.8 \mathrm{MHz}\right.$, fréquence centrale du traducteur). Par ailleurs, la zone focale est un cylindre centré au point focal de longueur $l=4 \lambda_{c} F / D$ et de largeur à $-6 \mathrm{~dB} d_{6}=\lambda_{c} F / D$ [7]. La plus grande profondeur de champ est donc obtenue pour la plus grande distance focale possible. A.N.: $F=94 \mathrm{~mm}, l=57 \mathrm{~mm}, d_{6}=5 \mathrm{~mm}$. La cible ne serait pas résolue par ce traducteur focalisé, alors qu'elle l'est ( $h \approx 3 \mathrm{~dB}$ pour $z=94 \mathrm{~mm}$ ) avec le traducteur plan de profil $\Gamma_{3}$. Pour des traducteurs focalisés à plus courte distance focale, la cible serait moins bien résolue (à cette fréquence centrale et pour ce diamètre de traducteur) qu'avec le traducteur plan de profil $\Gamma_{3}$. L'effet focalisant du traducteur plan avec pour profil $\Gamma_{3}$ est donc considérable.

a)

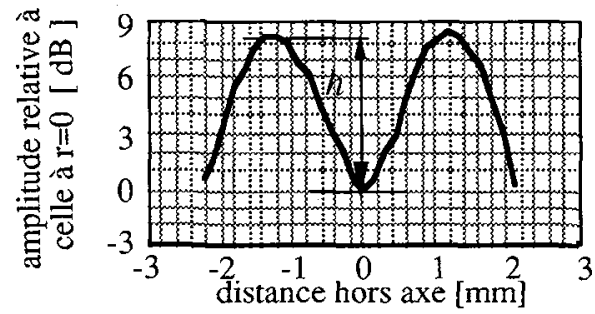

b)

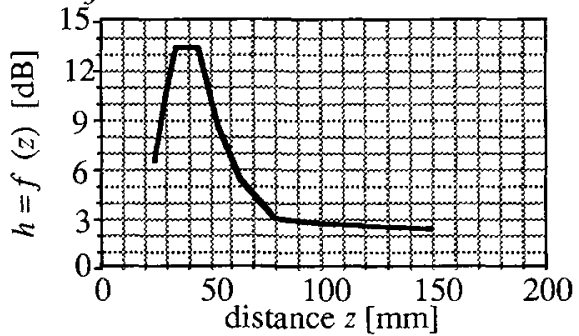

Fig. 4 - a) Échodynamique en fonction de la distance hors axe à $55 \mathrm{~mm}$ du traducteur et définition de $h$. -b) Variation de $h$ avec la distance au traducteur.

\subsection{Résolution axiale du traducteur à onde de bord}

La résolution axiale du traducteur de profil $\Gamma_{3}$ est moins bonne que celle du traducteur de profil $\Gamma_{2}$, mais meilleure que celle du traducteur uniforme. L'étude précise de cette résolution n'est pas présentée ici. 


\section{SENSIBIITÉ DES TRADUCTEURS NON-UNIFORMES.}

Les traducteurs uniformes conventionnels sont fabriqués pour maximiser la tansduction électro-acoustique. Dès lors, quelle que soit la méthode mise en œuvre pour modifier le profil du traducteur, l'énergie acoustique rayonnée par un traducteur non-uniforme est nécessairement plus faible que celle rayonnée par un traducteur uniforme. Cependant, il se peut, c'est le cas en champ proche, que l'énergie supplémentaire rayonnée par le traducteur uniforme soit source d'échos rendant l'interprétation difficile voire impossible. La discussion sur l'amplitude est donc plus subtile qu'il n'y parait. Pour pouvoir mener une étude quantitative, nous avons relevé, pour les trois profils considérés, l'amplitude crête à crête de l'écho revenant d'un petit disque de diamètre $2 \mathrm{~mm}$ sur 1'axe de symétrie (acoustique) en fonction de la distance. Les résultats présentés Fig. 5 donnent l'amplitude des échos obtenus avec les deux traducteurs modifiés (de profil $\Gamma_{2}$ et $\Gamma_{3}$ ) relative (en $\mathrm{dB}$ ) à celle des échos obtenus avec le traducteur uniforme $\left(\Gamma_{1}\right)$.

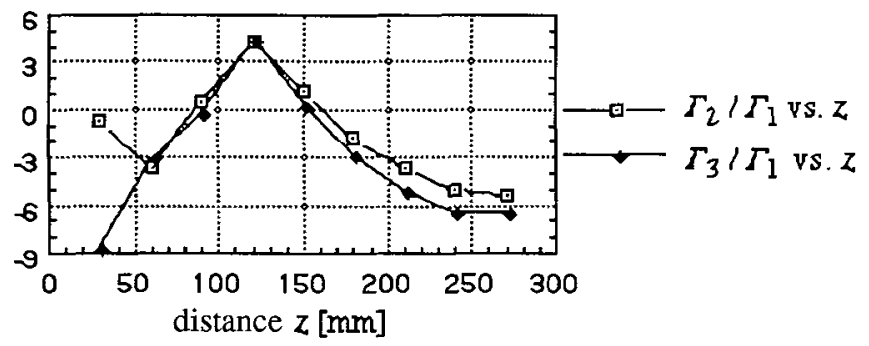

Fig. 5 - Amplitudes crête à crête des échos obtenus avec les traducteurs $\Gamma_{2}$ and $\Gamma_{3}$ relatives à celle obtenue avec $\Gamma_{1}$ en fonction de $z$.

En champ lointain, les deux courbes tendent asymptotiquement vers les valeurs $-5 \mathrm{~dB}$ pour $\Gamma_{2} / \Gamma_{1}$ et de $-7 \mathrm{~dB}$ pour $\Gamma_{3} / \Gamma_{1}$ proches des $-5.1 \mathrm{~dB}$ et $-7.1 \mathrm{~dB}$ que sont les valeurs relatives de leur surface active équivalente respective. En champ proche, i.e., pour $z<a^{2} v_{c} / c \approx 154 \mathrm{~mm}$, le rapport d'amplitude est éventuellement positif pour $\Gamma_{2}$ et $\Gamma_{3}$, i.e., la sensibilité est meilleure que celle du traducteur uniforme. Si l'on compare à nouveau le traducteur plan de profil $\Gamma_{3}$ avec un traducteur focalisé de même diamètre, il est clair que les sensibilités ne sont plus comparables: l'énergie du premier est concentrée le long de l'axe acoustique sur une grande profondeur et est rayonnée par une surface efficace plus faible, celle du second est concentrée le long de l'axe sur une zone de profondeur réduite et est rayonnée par toute la surface.

\section{RÉSUMÉ ET DISCUSSION.}

La forme du profil de source et sensibilité d'un traducteur a un effet considérable sur les mesures échographiques large bande. La comparaison des échos simulés et mesurés montre que les profils créés sont conformes à ceux souhaités. Les traducteurs modifiés ne nécessitent aucune électronique spécifique. Leur production industrielle est en étude (des prototypes industriels de traducteurs à profils modifiés selon les méthodes mises au point au laboratoire sont en cours d'évaluation). L'utilisation des tels traducteurs conduit à une simplification du champ incident aux défauts (application CND) et du champ reçu des défauts, d'autant plus que l'élément diffusant est dans le champ proche du traducteur. Cela est particulièrement vrai pour le traducteur de profil $\Gamma_{2}$ qui se comporte comme une source non-diffractante dans le champ proche. Pour le traducteur de profil $\Gamma_{3}$, la simplification est moins évidente. Son principal intérêt réside en son excellente résolution radiale sur une très grande profondeur de champ, résolution meilleure que celle des traducteurs focalisés classiques de même diamètre et même fréquence. Relativement à ces derniers, sa sensibilité est en revanche plus faible. Dans le champ lointain, i.e., lorsque les différentes contributions du traducteur atteignent la cible presque simultanément, les réponses obtenues sont similaires, quel que soit le traducteur utilisé, mais l'amplitude est proportionnelle à la surface active du traducteur, ce qui conduit à une moins bonne sensibilité des traducteurs modifiés que d'un traducteur conventionnel. Dans le champ proche, en plus du gain qualitatif de simplification des formes d'onde avec les traducteurs non-uniformes, on a éventuellement gain quantitatif de sensibilité.

[1] Lhémery A. et De Vadder D., "Réponses échographiques de cibles insonifiées par des traducteurs large bande à profil non-uniforme", 2 ème Congrès Français d'Acoustique Volume II, J. Roux Ed., Arcachon France avril 1992 (Les Éditions de Physique, Les Ulis, 1992) pp. 827-830.

[2] Weight J.P. and Hayman A.J., J. Acoust. Soc. Am.. 63 (1978) 396-404.

[3] Newberry B., Preischel M.and McKain J., Review of Progress in QNDE 11 (1992) 999-1004.

[4] Lhémery A., J. Acoust. Soc. Am. 90 (1991) 2799-2807.

[5] Lhémery A.and Raillon R., accepté pour publication dansJ. Acoust. Soc. Am.

[6] Brittain R.H. and Weight J.P., Ultrasonics 25 (1987) 100-106.

[7] AFNOR, NF A 09 - 330. 